\title{
A novel ingress node design for video streaming over optical burst switching networks
}

\author{
S. Askar,* G. Zervas, D. K. Hunter, and D. Simeonidou \\ School of Computer Science and Electronic Engineering, University of Essex, Colchester CO4 3SQ, UK \\ *skaske@essex.ac.uk
}

\begin{abstract}
This paper introduces a novel ingress node design which takes advantage of video data partitioning in order to deliver enhanced video streaming quality when using H.264/AVC codec over optical burst switching networks. Ns2 simulations show that the proposed scheme delivers improved video traffic quality without affecting other traffic, such as best effort traffic. Although the extra network load is comparatively small, the average gain in video PSNR was $5 \mathrm{~dB}$ over existing burst cloning schemes, with a maximum end-to-end delay of $17 \mathrm{~ms}$, and jitter of less than $0.35 \mathrm{~ms}$.
\end{abstract}

(C)2011 Optical Society of America

OCIS codes: (060.4250) Networks; (060.2330) Fiber optics communications.

\section{References and links}

1. B. Mukherjee, Optical Communications Networks (McGraw-Hill, New York, 1997).

2. M. Duser and P. Bayvel, "Analysis of a dynamically wavelength-routed optical burst switched network architecture,” J. Lightwave Technol. 20(4), 574-585 (2002).

3. Y. Chen, C. Qiao, and X. Yu, "Optical burst switching: a new area in optical networking research," IEEE Netw. 18(3), 16-23 (2004).

4. W. Liao and C.-H. Loi, "Providing service differentiation for optical-burst-switched networks," J. Lightwave Technol. 22(7), 1651-1660 (2004).

5. K. Chua, M. G, Y. Liu, and M. Phung, "Relative quality of service differentiation," in Quality of Service in Optical Burst Switched Networks, B. Mukherjee, ed. (Springer, New York, 2007).

6. A. Belbekkouche, A. Hafid, M. Gendreau, and M. Tagmouti, "Path-based QoS provisioning for optical burst switching networks,” J. Lightwave Technol. 29(13), 2048-2063 (2011).

7. W.-S. Park, M. Shin, H.-W. Lee, and S. Chong, "A joint design of congestion control and burst contention resolution for optical burst switching networks,” J. Lightwave Technol. 27(17), 3820-3830 (2009).

8. T. Venkatesh, A. Sankar, A. Jayaraj, and C. Murthy, "A complete framework to support controlled burst retransmission in optical burst switching networks," IEEE J. Sel Area Commun. 26(3), 65-73 (2008).

9. M. Waheed, "Experimental evaluation of VBR transport over optical burst switching network," in 2009 2nd IEEE International Conference on Computer Science and Information Technology (IEEE, 2009), pp. 623-627.

10. W. Zhang, X. Hong, Y. Yin, H. Jiang, L. Liu, H. Guo, J. Wu, and J. Lin, "Experimental investigation of high definition video clips (HDVC) streaming over OBS Networks," in 35th European Conference on Optical Communication, 2009. ECOC '09 ( 2009), pp. 1-2.

11. D. Pevac, I. Petrovic, and R. Bojovic, "The possibility of application the optical wavelength division multiplexing network for streaming multimedia distribution," in 2011 IEEE EUROCON-International Conference on Computer as a Tool (EUROCON), (IEEE, 2011), pp. 1-4.

12. F. Espina, M. Izal, D. Morató, and E. Magaña, "Performance analysis of OBS edge nodes for video streaming," in ICCCN 2009. Proceedings of 18th Internatonal Conference on Computer Communications and Networks, 2009 (2009), pp. 1-6.

13. M. Izal, F. Espina, D. Morató, and E. Magaña, "Ingress traffic classification versus aggregation in video over OBS networks," in 2nd Workshop on Multilayer Networks (2010), pp. 47-52.

14. S. Askar, G. Zervas, D. K. Hunter, and D. Simeonidou, "Classified cloning for QoS provisioning in OBS networks," in 2010 36th European Conference and Exhibition on Optical Communication (2010), pp. 1-3.

15. S. K. Askar, G. S. Zervas, D. K. Hunter, and D. Simeonidou, "Adaptive classified cloning and aggregation technique for delay and loss sensitive applications in OBS networks," in Optical Fiber Communication Conference, OSA Technical Digest (CD) (Optical Society of America, 2011), paper OThR4.

16. Cisco, “Cisco Visual Networking Index: Forecast and Methodology, 2010-2015” (2011), http://www.cisco.com/en/US/solutions/collateral/ns341/ns525/ns537/ns705/ns827/white_paper_c11481360_ns827_Networking_Solutions_White_Paper.html.

17. JVT Reference Software Version JM16 1; see http://iphome.hhi.de/suehring/tml/.

\#155698 - \$15.00 USD

(C) 2011 OSA
Received 5 Oct 2011; revised 2 Nov 2011; accepted 8 Nov 2011; published 18 Nov 2011 12 December 2011 / Vol. 19, No. 26 / OPTICS EXPRESS B191 
18. J. Klaue, B. R. Rathke, and A. Wolisz, "EvalVid-a framework for video transmission and quality evaluation," in Computer Performance Evaluation. Modelling Techniques and Tools, Vol. 2794/2003 of Lecture Notes in Computer Science (Springer, 2003.), pp. 255-272.

19. X. Huang, V. M. Vokkarane, and J. P. Jue, "Burst cloning: a proactive scheme to reduce data loss in optical burst-switched networks,” in 2005 IEEE International Conference on Communications, 2005. ICC 2005, (IEEE, (2005), Vol. 3, pp. 1673-1677.

\section{Introduction}

The tremendous growth of Internet traffic, and the corresponding increase in link bandwidth, have motivated deployment of optical wavelength division multiplexing (WDM) communication in many telecommunications backbone networks [1]. Three switching schemes have been proposed for WDM networks, namely optical circuit switching, optical packet switching, and optical burst switching (OBS). Optical burst switching is envisioned as the technology that will support growing future bandwidth needs efficiently, as in many ways it combines the merits of OCS and OPS while overcoming their shortcomings [2,3]. However, with the prevalence of triple-play services (i.e., data, voice, and video) and web-based multimedia applications, Quality of Service (QoS) differentiation has become a crucial issue for future OBS networks intended for practical deployment [4-6].

Many mechanisms have been proposed and/or implemented to reduce the loss rate in OBS networks; they may be categorized as either loss minimization or loss recovery methods. Loss recovery mechanisms are either reactive (retransmission) or proactive (forward error correction, composite burst assembly, 1+1 protection, cloning) while loss minimization approaches can be divided into contention resolution (fiber delay line, deflection routing, wavelength conversion, segmentation) and contention avoidance (admission control, load balancing, serialization) schemes. Contention avoidance aims to prevent contention from taking place while contention resolution schemes seek to resolve contention that already exists [7].

Contention resolution mechanisms are the most commonly discussed, however their implementation is hindered by many factors such as: 1) the immaturity of wavelength conversion and the cost of implementing it, 2) the physical size and thermal instability of fiber delay lines, coupled with the fact that they merely offer fixed delays which generally reduce channel utilization because they generate voids between scheduled bursts, 3) the routing loops which are often introduced by deflection routing as well as the possibility of insufficient offset time for rerouted bursts, 4) the complexity of implementing burst segmentation. Reactive loss recovery can retransmit bursts in the event of contention, however it requires very large buffers at the ingress node, making it impractical in MAN's and WAN's, while also increasing delay and jitter [8].

Research into video over OBS networks [9-13] appears to be focusing more on evaluating and investigating performance aspects, perhaps when mixing video with other traffic types, rather than proposing a feasible scheme which can demonstrably offer improved quality of video delivery by overcoming fundamental difficulties with OBS. We have already proposed a new scheme to enforce QoS parameters such as loss, delay, and jitter for video applications $[14,15]$, but although it performs very well in provisioning video traffic which constitutes $30 \%$ or less of the total, performance degrades when the percentage is higher. It is expected that video will constitute $61 \%$ of overall consumer traffic by 2015 [16]. This paper introduces a novel scheme for video delivery over OBS, and shows through simulation that it can accommodate this traffic increase. Extensive simulations were conducted to evaluate the proposed scheme under a range of scenarios which consider the effect of increased video traffic.

The remainder of this paper is organized as follows: Section 2 introduces the new technique. Section 3 evaluates its performance, while Section 4 concludes the paper.

\#155698 - \$15.00 USD

(C) 2011 OSA
Received 5 Oct 2011; revised 2 Nov 2011; accepted 8 Nov 2011; published 18 Nov 2011 12 December 2011 / Vol. 19, No. 26 / OPTICS EXPRESS B192 


\section{The proposed scheme}

It has been shown that the Classified Cloning Scheme (CCS) is effective in reducing the burst loss rate, especially when the proportion of video traffic is light to medium. It was shown that the performance of CCS declines as the percentage of video traffic is increased from $10 \%$ to $30 \%$ [15]. In this paper, we seek to maintain performance while minimizing the increase in load due to burst duplication by capitalizing on the importance of certain video packets obtained when encoding video data partitioning with a H.264/AVC codec. Henceforth we will refer to proposed scheme as the Enhanced Scheme (ES).

The routing module in the ingress node sends each incoming packet to a specific aggregation queue based on its destination address and priority. Packet classification at the ingress node is used to identify packets which form video traffic. A novel ingress node design - the ES ingress node-is shown in Fig. 1. The top buffer (Buffer 0) is allocated for Best Effort (BE) traffic, while two further buffers are allocated for video traffic. Writing to buffers 1 and 2 is enabled when receiving video traffic (i.e. the "video_" signal is activated) as shown in the Figure. To avoid adding any extra delay, the two buffers effectively function as one buffer. Having two buffers permits switching over the process of storing " $A$ " packets among the second and third buffers (Buffer 1 and Buffer 2) whenever a new burst is generated. Every time a burst is generated, the value of the "status" signal is toggled (using a T flip-flop which is not shown). If the "status" signal is " 0 " then (as shown in the Figure), Buffer 1 receives the incoming traffic (it has to be video as defined by the writing pin) while Buffer 2 receives " $A$ " packets from the same video stream. When Buffer 1 generates a burst, the "status" signal becomes " 1 ". The incoming traffic now enters Buffer 2 (which has already been partially filled by "A" packets) while "A" packets from the current video go to Buffer 1 .

The network abstraction layer (NAL) provides an interface between the video coding layer and the network layer, so that the network layer can provision some packets preferentially, depending on their type. With H.264/AVC codec, video data partitioning is almost universally used, in which case each video frame is identified as being an "A" packet, a " $\mathrm{B}$ " packet, or a "C" packet. If data partitioning is not used, the ES ingress node will still carry the video traffic, although without any improvement in QoS. "A" packets are the most important because they contain the addresses of the other two packet types, but surprisingly they are the smallest in size, which is the motivation for this work. Duplicating them does not contribute as much to the network load as duplicating " $\mathrm{B}$ " or " $\mathrm{C}$ " packets. Moreover, if some video packets are lost, then the video quality depends on the type of packet-losing " $\mathrm{B}$ " or " $\mathrm{C}$ " packets has less effect than losing " $A$ " packets. " $A$ " packets are duplicated and sent in another burst which does not carry "B" and "C" packets, in order to enhance recovery (video reconstruction at the receiver side), which will be demonstrated in the following section. Although the NAL unit may be encrypted, this applies to the video data only, and the slice header (which identifies the packets as "A", "B" or "C") is in practice never encrypted.

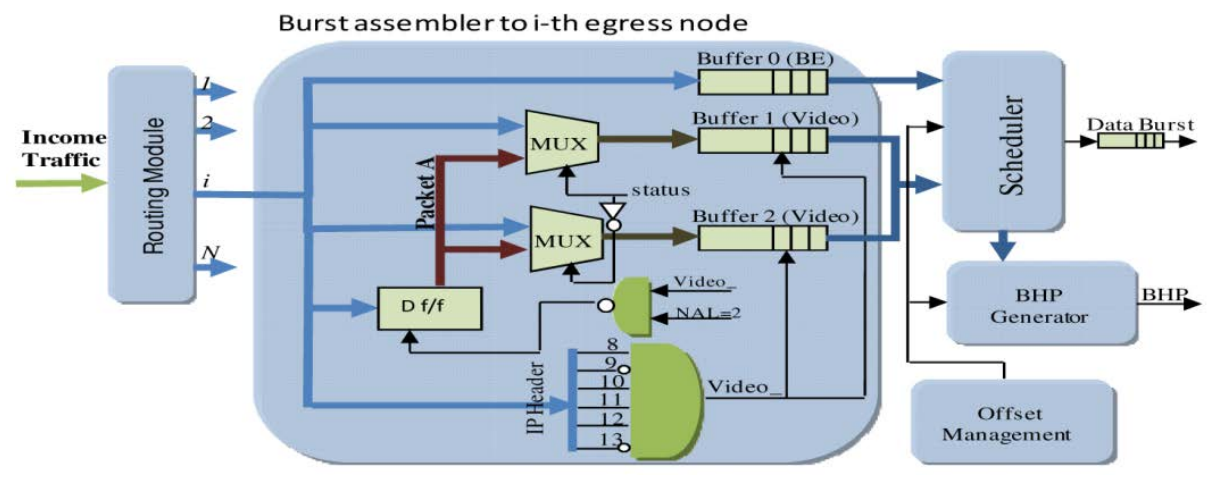

Fig. 1. ES ingress node

\#155698 - \$15.00 USD

(C) 2011 OSA
Received 5 Oct 2011; revised 2 Nov 2011; accepted 8 Nov 2011; published 18 Nov 2011 12 December 2011 / Vol. 19, No. 26 / OPTICS EXPRESS B193 


\section{Simulation results}

The ns-2 simulator was used to evaluate the proposed scheme on a 14-node NSF network topology (Fig. 2(a)) where each link had sixteen 10 Gbps channels in each direction. The load was distributed among the ingress-egress nodes uniformly so that when the proportion of video traffic was for example $10 \%$, all ingress nodes sent $10 \%$ video traffic and $90 \%$ BE traffic. Burst aggregation was based upon a size threshold, in which the maximum burst size was set to 500 KBytes. Size threshold was chosen to avoid performance degradation in case of utilizing time out aggregation technique with multiple burstifiers [13]. The core nodes were bufferless and the wavelength continuity constraint was observed. The OBS control plane supported JET (Just Enough Time). Data bursts were scheduled with the LAUC-VF (Latest Available Unscheduled Channel with Void Filling) channel scheduling protocol with control bursts scheduled by LAUC. All routes were established by a shortest path routing algorithm with the number of hops as the metric.

Five different 10 -second CIF $(352 \times 288)$ standard test sequences were encoded using H.264/AVC (Advanced Video Coding) reference software JM16.1 [17] with 30 frames per second. Video data partitioning was implemented in the encoding process in order to facilitate priority provisioning over the OBS network through duplication at the ingress node of "A" packets, which are the most important. The encoded H.264 videos were then converted into trace files which were fed into the OBS network. The simulations were run many times to obtain 99\% confidence intervals, and for each run, the received H.264 file was derived from the trace file which was produced. The H.264 videos were then decoded to obtain a YUV video format. The resulting PSNR (Peak Signal to Noise Ratio) was measured by using the EvalVid framework [18] to compare this new file with the original lossless YUV video.

As mentioned earlier, "A" packets are smaller than other packets, although they are crucial to the quality of the resulting video. Because the aim of this paper is to minimize the increase in load when the proportion of video traffic increases, three different scenarios were investigated in which the proportion of video traffic was $10 \%, 30 \%$, and $50 \%$ respectively. Figure 2(b) compares the Basic Cloning Scheme (BCS) [19], CCS, and ES. Figure 2(b) shows that for BCS and CCS, changing the type of video sequence (Akiyo, Stefan, Paris, Foreman, and Mobile) doesn't affect the increase in load - this is because the videos are sent at a similar rate (1 Mbps for each video sequence). However when applying ES, the type of video does have a slight effect because each video sequence has a different proportion of " $A$ " packets, therefore the duplication of "A" packets varies slightly among different videos as shown in Fig. 2(b).
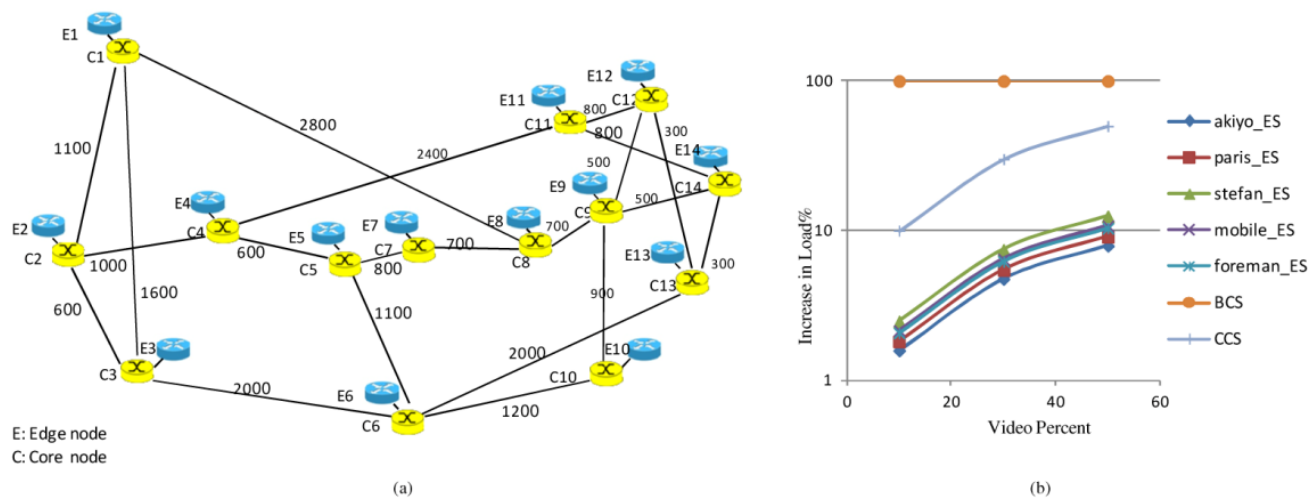

Fig. 2. (a) 14-node NSF network topology (b) Extra load added due to applying different schemes

Figures 3(a), (b), and (c) show the PSNR values for the Akiyo, Stefan, and Mobile video sequences respectively. As the proportion of video traffic increases, the added load increases,

\#155698 - \$15.00 USD

(C) 2011 OSA
Received 5 Oct 2011; revised 2 Nov 2011; accepted 8 Nov 2011; published 18 Nov 2011 12 December 2011 / Vol. 19, No. 26 / OPTICS EXPRESS B194 
thus increasing the loss rate. Figures 3(a), (b), and (c) compare the PSNR values when streaming a video without ES against the case where ES was implemented with a video percentage equivalent to $50 \%$ of the total traffic which is the worst-case scenario for the ES scheme. Figures 4(a), (b), and (c) compare PSNR values with 10\%, 30\%, and 50\% video traffic, with video traffic from Akiyo, Stefan, and Mobile video sequences. Figures 3(a), (b), and (c) show considerable improvement in PSNR for the different video sequences. Moreover, the proposed scheme exhibits very robust performance; it clearly outperforms other schemes in the worst-case scenario, when video composes $50 \%$ of the total traffic. Figure 4 compares the PSNR values for three video sequences when the video comprises $10 \%, 30 \%$, and $50 \%$ of the total traffic, showing that as the percentage increases there is little effect on the received video quality. Clearly the objective has been attained of alleviating the performance degradation as the proportion of video traffic increases.
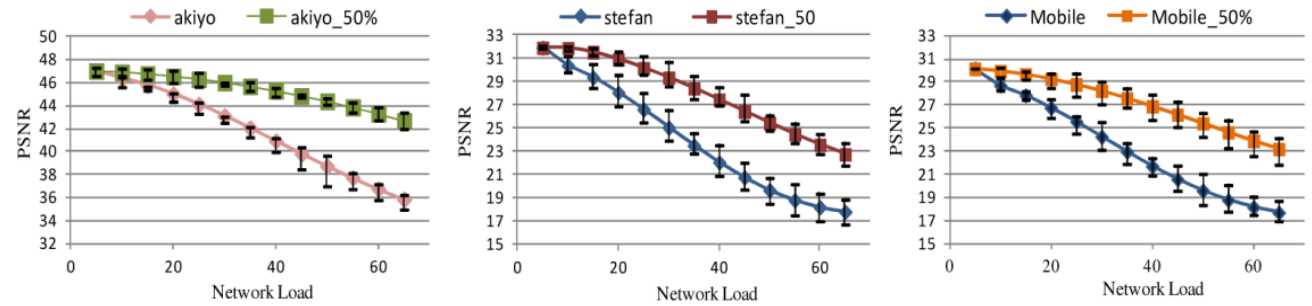

Fig. 3. Video quality, regular streaming versus worst-case ES scheme video streaming for (a) Akiyo (b) Stefan (c) Mobile video sequences

Figures 5(a), (b), and (c) show the loss rate of Akiyo, Stefan, and Mobile video sequences respectively. With ES, there is a slight increase in the loss rate because of the increased network load, however bear in mind that this loss is distributed across the whole streamed video which includes the original video plus the duplicated "A" packets. However as shown in Fig. 5, video streamed with ES will has a much lower loss rate for "A" packets, which results in improved video quality. The way duplication of "A" packets is carried out plays a
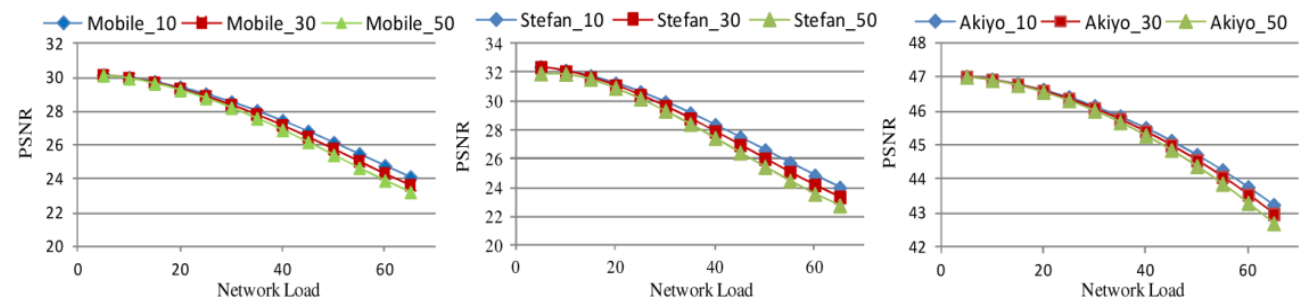

Fig. 4. Video quality comparison of (a) Akiyo, (b) Stefan, (c) Mobile video sequences in three scenarios; $10 \%, 30 \%$, and $50 \%$ video rates ES streaming

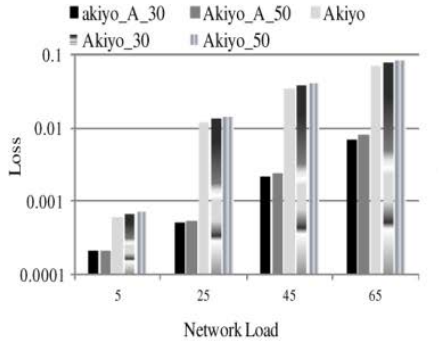

(a)

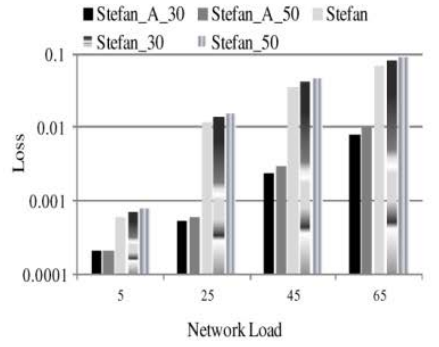

(b)

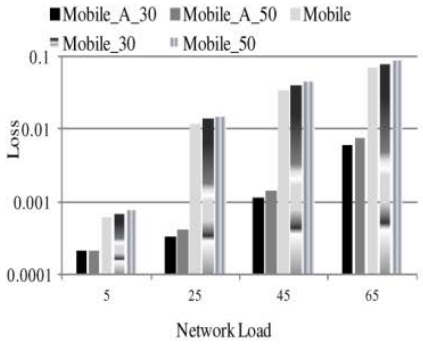

(c)

Fig. 5. Video loss rate comparison; regular streaming, 30\% ES, and 50\% ES streaming for three different video sequences (a) Akiyo (b) Stefan (c) Mobile

\#155698 - \$15.00 USD

(C) 2011 OSA
Received 5 Oct 2011; revised 2 Nov 2011; accepted 8 Nov 2011; published 18 Nov 2011 12 December 2011 / Vol. 19, No. 26/ OPTICS EXPRESS B195 


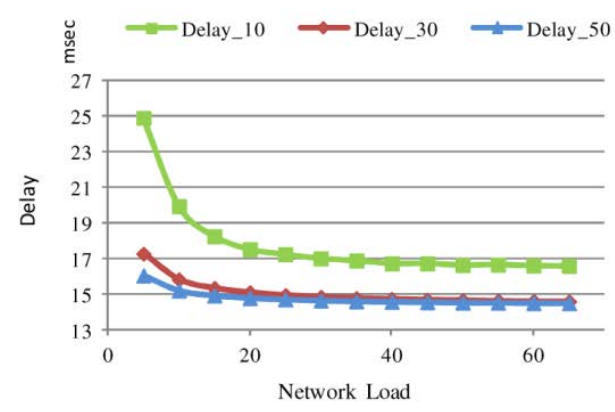

(a)

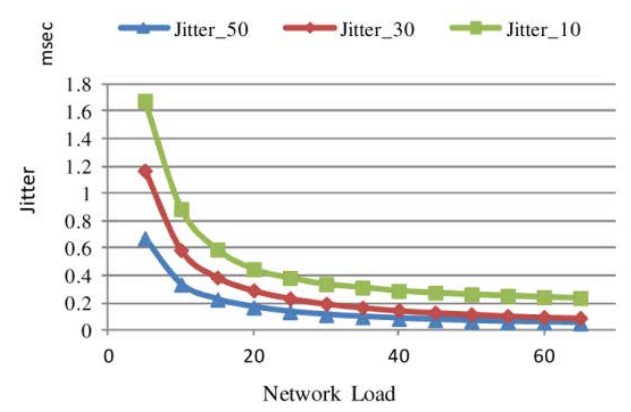

(b)

Fig. 6. (a) Average End to End Delay and (b) Average Jitter

very important role in reconstructing a video with higher quality. If one burst is dropped video packets may be lost, however the video can still be reconstructed because its complement is in another burst (due to the use of Buffer 1 and Buffer 2). Figure 6(a) shows the average ETE delay for video traffic from the five sequences, demonstrating that with the size threshold aggregation technique, ETE delay reduces as incoming traffic increases.

Figure 6(b) shows the mean jitter value for the video sequences. Like ETE delay, jitter increases as the proportion of video traffic increases. However, the maximum average jitter is $1.7 \mathrm{~ms}$ when the proportion of video traffic is $10 \%$ and the network load is $5 \%$. At higher loads and with the same proportion of video traffic, jitter stabilizes and settles to below 0.35 ms.

\section{Conclusions}

This paper presented a novel ingress node design which delivers enhanced streamed video across OBS networks. The proposed scheme is called the Enhanced Scheme, in which the most important packets arising from video data partitioning encoding are switched between two buffers in the ingress node in order to facilitate better video reconstruction.

Extensive ns-2 simulations were conducted to evaluate the Enhanced Scheme, showing its efficiency where an average improvement of $5 \mathrm{~dB}$ in video quality was obtained in the worstcase scenario with medium and high network loads. The proposed scheme improves the quality of high-priority traffic (i.e. video traffic) without significantly affecting the best effort traffic loss rate, which increased by only $12 \%$ in the worst-case scenario. The end-to-end delay was below $17 \mathrm{~ms}$ with a network load of greater than $20 \%$ while the average jitter value was less than $0.35 \mathrm{~ms}$ under the same network load.

Future work may evaluate the suggested scheme for scalable video streaming, in which a H.264/SVC codec will be used instead of the H.264/AVC. Instead of duplicating "A” packets at the ingress node, duplication of the base layer will be investigated, and performance will be evaluated in that case.

\section{Acknowledgments}

This work was supported by the EC through IST STREP project MAINS (INFSO-ICT247706).

\#155698 - \$15.00 USD

(C) 2011 OSA
Received 5 Oct 2011; revised 2 Nov 2011; accepted 8 Nov 2011; published 18 Nov 2011 12 December 2011 / Vol. 19, No. 26 / OPTICS EXPRESS B196 